\title{
Demographic and Clinical Characteristics of Individuals with Mild Cognitive Impairment Related to Grade of Alcohol Consumption
}

\author{
Theofanis Tsevis $^{a, b} \quad$ Eric Westman ${ }^{a} \quad$ Konstantinos Poulakis ${ }^{a} \quad$ Olof Lindberg $^{a}$ \\ Atef Badji $^{a}$ b Dorota Religa ${ }^{a, b}$ Lars-Olof Wahlund ${ }^{a, b}$ \\ aDivision of Clinical Geriatrics, Center for Alzheimer Research, Department of Neurobiology, Care Sciences and

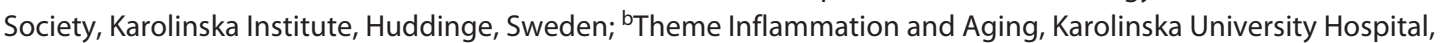 \\ Huddinge, Sweden
}

\section{Keywords}

Alcoholic dementia · Biomarkers · Cognitive assessment . Mild cognitive impairment

\begin{abstract}
Introduction: While alcohol overconsumption is regarded as a risk factor for Alzheimer's disease, the specific relationship between alcohol consumption and cognitive impairment remains unclear and poorly understood. Our primary objective is to investigate whether alcohol consumption is associated with lower cognitive performance at an early phase of the development of cognitive impairment (mild cognitive impairment $[\mathrm{MCl}]$ stage) and second to present the clinical and demographic characteristics depending on the grade of alcohol consumption. Methods: This is a cross-sectional observational study, including 251 subjects with the diagnosis $\mathrm{MCl}$, having caregiving contact with Memory Clinic, Karolinska University Hospital, under year 2015. We compared subgroups with different levels of alcohol consumption, concerning social parameters, cognitive, radiological, laboratory profile as well as comorbidities and burden of drugs. Results: Mini-mental State Examination score was not associated with alcohol consumption. Light to moderate drinkers were significantly higher educated. There were significantly more subjects using antianxiety medications among
\end{abstract}

heavy drinkers in comparison with light to moderate drinkers. Finally, never/rare drinkers had significantly lower levels of erythrocyte mean corpuscular volume in their blood tests. Discussion/Conclusion: Alcohol consumption was not correlated with a more pronounced cognitive deficit or a distinct clinical severity at an early stage of cognitive impairment apart from higher usage of antianxiety medications. We are planning to follow up all individuals to ascertain if heavy drinkers have a different outcome compared with the other groups.

(C) 2021 The Author(s)

Published by S. Karger AG, Basel

\section{Introduction}

Long-term and extensive alcohol consumption can lead to reduced cognitive performance $[1,2]$ and is identified as one of the most important risk factors for dementia [3-5]. Contrastingly, the specific relationship between alcohol consumption and cognitive performance is inadequately defined and poorly explained $[6,7]$. One of the major problems is the varying elucidation of alcohol amounts among the epidemiological studies in the literature, which is partly related to broad discrepancies in the size of standard drink between countries and continents

Correspondence to:

Theofanis Tsevis, theofanis.tsevis@ki.se

karger@karger.com www.karger.com/dem

Karger $\stackrel{\text { ' }}{5}$

BOPEN ACCESS
(C) 2021 The Author(s)

Published by S. Karger AG, Basel

This is an Open Access article licensed under the Creative Commons Attribution-NonCommercial-4.0 International License (CC BY-NC) (http://www.karger.com/Services/OpenAccessLicense), applicable to the online version of the article only. Usage and distribution for commercial purposes requires written permission. 


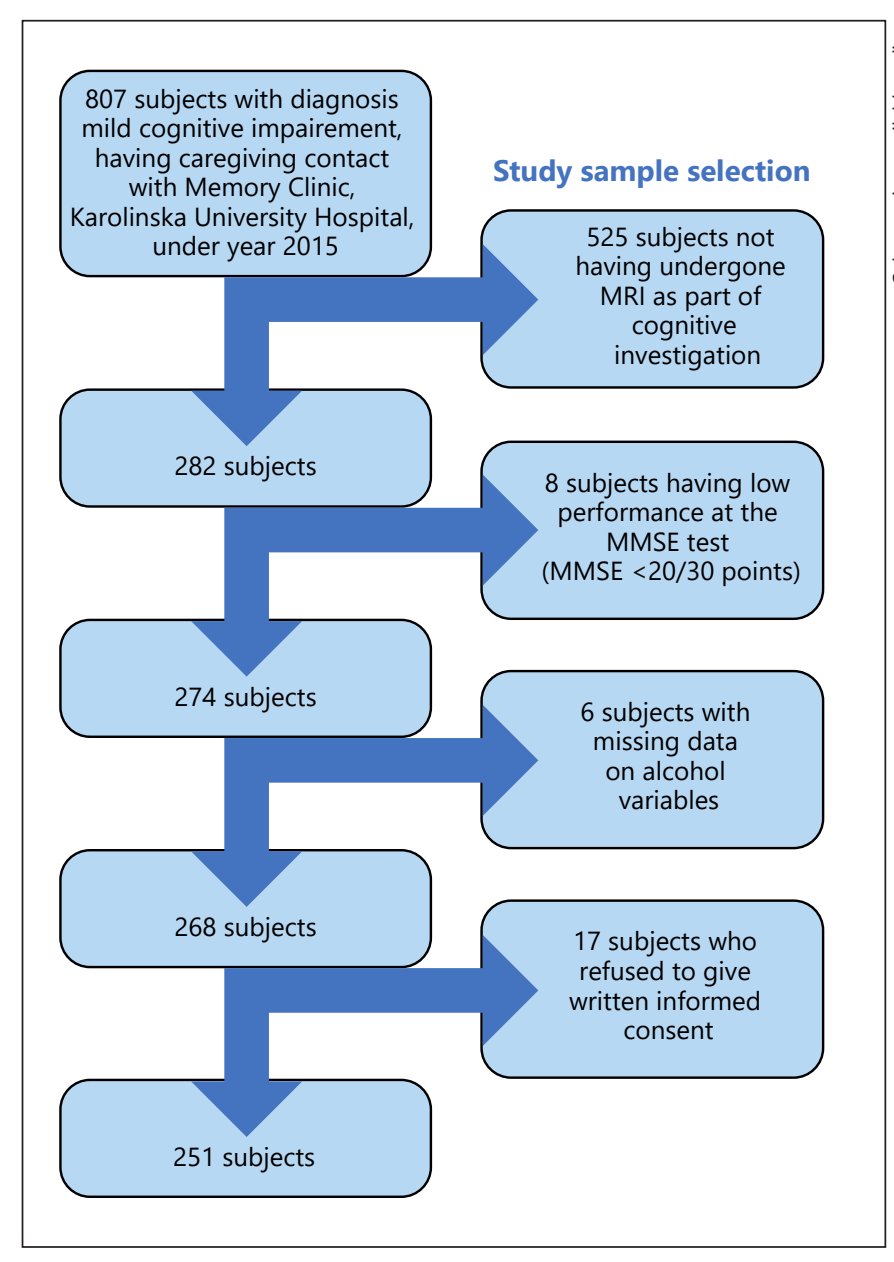

Fig. 1. Study sample selection. MCI, mild cognitive impairment; MRI, magnetic resonance imaging; MMSE, Mini-mental State Examination.

[8]. In Europe, the size of a standard drink ranges by $250 \%$ according to a review study [9]. Thus, it is challenging to specify low-risk drinking levels. Quantity and frequency of alcohol consumed determine whether the effects of alcohol are positive or negative [10]. Individuals with alcohol intake over 240 gr/week have higher risk for structural damages, especially in hippocampus [11]. In addition, heavy consumption for elderly individuals $(>65)$ has been defined as $>100 \mathrm{~g} /$ week in the literature $[12,13]$. On the other hand, several epidemiological studies assert that low to moderate alcohol consumption can be beneficial for developing cognitive impairment, particularly Alzheimer's disease (AD) $[14,15]$.

Our primary objective is to investigate whether alcohol consumption is associated with lower cognitive performance as well as significantly altered biomarkers in cerebrospinal fluid (CSF) and on magnetic resonance imaging (MRI) at an early phase of the development of cognitive impairment (mild cognitive impairment [MCI] stage). Our secondary aim is to present the clinical and demographic characteristics of subjects with MCI by comparing subgroups with different levels of alcohol consumption.

\section{Materials and Methods}

\section{Participants and Cohort}

We have selected our material from subjects from Stockholm County who have undergone cognitive investigation at the Memory Clinic, Karolinska University Hospital. Cognitive assessment consists of anamnestic interview, somatic examination, cognitive testing with Mini-mental State Examination (MMSE), neuropsychological examination, radiological control, and standard blood and CSF tests. The approximate duration of cognitive assessment procedure is 6-12 months.

Our cohort comprised subjects with MCI diagnosis, which corresponds to an early stage of cognitive impairment $[16,17]$. We initially selected all subjects $(n=807)$ with MCI diagnosis (ICD-10 code; F067) having caregiving contact with the Memory Clinic under year 2015.

We excluded subjects not having undergone MRI, having low performance ( $<20$ points) at the MMSE or incomplete information on alcohol consumption and finally those who refused to give written informed consent, which resulted in 251 subjects (shown in Fig. 1). 142 subjects had a cognitive investigation in 2015, 73 in 2014, 26 in 2013, 6 in 2012, and 4 in 2011. This study has a crosssectional observational design.

Cognitive Assessment

Cognitive function is rated by the Swedish version of MMSE $[18,19]$.

\section{Blood and CSF Tests}

Blood tests have been performed in a clinical context, where each subject was studied separately and included levels of hemoglobin, mean corpuscular volume (MCV), kidney function/glomerular filtration rate, albumin, thyroid-stimulating hormone, aspartate transaminase, alanine transaminase, Natrium, calcium, lipids (total-, high-density lipoprotein [cholesterol] and low-density lipoprotein [cholesterol]), homocysteine.

CSF samples included biomarkers for AD: tau protein, phosphorylated tau protein, and beta-amyloid. Prodromal AD defined by low levels of beta-amyloid is equally distributed among groups.

\section{Magnetic Resonance Imaging}

MRI scanners with $1.5 \mathrm{~T}$ or $3 \mathrm{~T}$ magnets were used, and $\mathrm{T} 1$ weighted MR images were acquired. All MRI scans were assessed by experienced radiologists in a clinical context. We used standardized visual rating scales $[20,21]$. For assessing medial temporal atrophy (MTA), the Scheltens scale was used [22], which is a scale between 0 and 4 points [23]. For cortical atrophy, we used the global cortical atrophy scale, which is a scale between 0 and 3 points [24], while assessment of white matter hyperintensities was 
conducted according to the Fazekas scale, which is a scale between 0 and 3 points $[20,25]$.

\section{Medications and Comorbidities}

Patients reported their medications and relevant chronic diseases in interview with the doctor during their visit at Memory Clinic. Each medication or comorbidity is dichotomized as having it or not.

\section{Demographic Factors}

Included demographic factors were age, sex, marital status (being married or not), educational level (elementary school, high school, and university), smoking (no smokers and current smokers), country of origin (Swedish-born and foreign-born subjects having immigrated to Sweden during adulthood), and body mass index (BMI) (online suppl. Table 1; for all online suppl. material, see www.karger.com/doi/10.1159/000519736).

\section{Measures of Alcohol Use}

All subjects have answered a health declaration questionnaire and participated in structured interviews performed by physicians during the first visit at the clinic regarding quantity and frequency of their weekly as well as lifetime alcohol consumption. Each beverage type was presented in the questionnaire as a unit which corresponds to a Swedish standard drink which contains $12 \mathrm{gr}$ of pure alcohol. According to the gender-specific Swedish guidelines, moderate consumption is defined as up to 9 Swedish standard drinks per week for women and 14 Swedish standard drinks per week for men [26]. No age-specific recommendations were found. Weekly alcohol consumption is categorized as follows: (a) no alcohol consumption or less than one Swedish standard drink, without having former alcohol overconsumption (Group N), (b) 1 to 9 Swedish standard drinks for women and 1 to 14 Swedish standard drinks for men, without having former alcohol overconsumption (Group LM), and (c) > 9 Swedish standard drinks for women, > 14 Swedish standard drinks for men, or/and former alcohol overconsumption according to their medical records (Group $\mathrm{H}$ ).

\section{Statistical Analysis}

Continuous and interval data are presented as means and standard deviations, while categorical data are presented as percentages and frequencies except for the MRI rating scales which are presented as median and interquartile ranges. Differences in nominal variables were tested with the $\chi^{2}$ test. Likelihood ratio is presented when assumptions of $\chi^{2}$ test were violated. Differences in continuous variables were tested with the Kruskal-Wallis nonparametric test. General linear model analysis was performed to evaluate association of alcohol consumption with cognitive function (MMSE score). Analyses were conducted using IBM SPSS Statistics 24 . The method of two-tailed test was used. $p$ values $<0.05$ were considered statistically significant.

\section{Results}

\section{Demographic and Clinical Variables}

Demographic and clinical data of all subjects included in the study as well as comparisons between groups were analyzed (Table 1, online suppl. Tables 2,3 ). Low to moderate drinkers were married in greater proportion and higher educated in comparison with the other groups. Moreover, they had lower BMI compared with never and rare drinkers. A greater proportion of men than women were heavy drinkers. There were significantly more smokers and more subjects using antianxiety medications among heavy drinkers in comparison with low to moderate drinkers. Never and rare drinkers had significantly lower proportion of Swedish origin and significantly lower levels of MCV in blood testing. No significant differences were identified regarding MRI rating scales and $\mathrm{AD}$-related biomarkers in CSF.

\section{Cognitive Test - MMSE}

A 2-way ANCOVA was conducted to compare the main effects of alcohol consumption and education as well as their interaction effects on MMSE score. Age was included in the analysis as covariate. The main effect of alcohol consumption yielded an effect size of 0.010 , indicating that $1 \%$ of the variance in MMSE score was explained by differences in alcohol consumption $(\mathrm{F}(2,213)$ $=1.103, p=0.334)$. The main effect of education yielded an effect size of 0.059 , indicating that $5.9 \%$ of the variance in MMSE score was explained by education $(F(2,213)=$ $6.665, p=0.002)$. The interaction effect was not significant $(\mathrm{F}(4,213)=1.345, p=0.254)$, indicating that there was no interaction effect between alcohol consumption and education. The covariate, age, was not significantly related to MMSE score $(\mathrm{F}(1,213)=2.277, p=0.133)$.

Group LM had marginally higher MMSE score (Adj M $=26.849, S E=0.285)$ in comparison with the other groups (Table 1). However, that score did not differ significantly from those acquired by either of the other groups (online suppl. Table 4).

\section{Discussion}

\section{Cognitive Functioning}

MMSE score does not differ significantly between our MCI groups divided according to the level of alcohol consumption. Only education had a significant main effect in our covariance analysis. These findings should be interpreted with caution, taking into consideration the complexity and the multifactorial character of alcohol effect on cognition. The results can be influenced by the fact that $\mathrm{H}$-group consists mainly of individuals with relatively low grade of high consumption. High alcohol consumption ( $\geq 36 \mathrm{~g} /$ day), particularly in male subjects, was 
Table 1. MMSE score and demographic and clinical characteristics

\begin{tabular}{|c|c|c|c|c|c|}
\hline Variables & All $(n=251)$ & Group N $(n=93)$ & Group LM $(n=104)$ & Group H $(n=54)$ & $p$ value \\
\hline \multicolumn{6}{|l|}{ Demographic data } \\
\hline Gender (women) ${ }^{\mathrm{b}, \mathrm{c}}, \%$ & 51.8 & 62.4 & 53.8 & 29.6 & 0.002 \\
\hline Age & $62.44(9.610)$ & $61.05(10.090)$ & $63.98(9.796)$ & $61.87(8.002)$ & 0.060 \\
\hline Education (university) $)^{a, c}, \%$ & 36.4 & 30.0 & 50.5 & 22.6 & 0.009 \\
\hline Smoking (smokers) ${ }^{c}, \%$ & 14.9 & 13.0 & 10.7 & 26.4 & 0.032 \\
\hline $\mathrm{BMI}^{\mathrm{a}}$ & $26.32(4.48)$ & $27.49(4.57)$ & $25.11(3.96)$ & $26.67(4.77)$ & 0.005 \\
\hline Marital status (partner) ${ }^{a, c}, \%$ & 63.3 & 50.5 & 81.7 & 50.0 & 0.000 \\
\hline Country origin (Sweden) $)^{a, b}, \%$ & 65.7 & 51.6 & 74.0 & 74.1 & 0.002 \\
\hline \multicolumn{6}{|l|}{ MMSE } \\
\hline Estimated MMSE means & 26.536 & $26.267(0.269)$ & $26.849(0.285)$ & $26.492(0.357)$ & 0.334 \\
\hline \multicolumn{6}{|l|}{ MRI rating scales } \\
\hline MTA, right & $1(1)$ & $1(2)$ & $1(1)$ & $1(1)$ & 0.624 \\
\hline MTA, left & $1(1)$ & $1(2)$ & $1(1)$ & $1(1)$ & 0.174 \\
\hline GCA & $1(1)$ & $1(1)$ & $1(1)$ & $1(1)$ & 0.174 \\
\hline WMH (Fazekas) & $1(2)$ & $1(1)$ & $1(1)$ & $1(1)$ & 0.345 \\
\hline \multicolumn{6}{|l|}{ CSF tests } \\
\hline Beta-amyloid & 736.27 (282.050) & 757.47 (303.509) & 743.28 (254.699) & 685.67 (304.987) & 0.717 \\
\hline Tau & 340.15 (201.912) & $347.41(205.923)$ & $332.64(186.102)$ & $345.13(232.801)$ & 0.949 \\
\hline Phospho-tau & $50.83(24.494)$ & $52.92(26.209)$ & $49.51(22.301)$ & $50.37(26.714)$ & 0.949 \\
\hline \multicolumn{6}{|l|}{ Blood tests } \\
\hline Hemoglobin & 139.09 (13.025) & $136.90(13.737)$ & $141.46(11.214)$ & $138.38(14.370)$ & 0.286 \\
\hline Albumin & $38.37(3.367)$ & $37.79(3.514)$ & $38.92(3.230)$ & $38.25(3.278)$ & 0.412 \\
\hline $\mathrm{Na}$ & $140.42(2.586)$ & $140.60(2.689)$ & $140.32(2.381)$ & $140.30(2.801)$ & 0.551 \\
\hline AST & $0.44(0.159)$ & $0.45(0.192)$ & $0.42(0.126)$ & $0.45(0.158)$ & 0.702 \\
\hline ALT & $0.44(0.264)$ & $0.45(0.282)$ & $0.43(0.276)$ & $0.43(0.212)$ & 0.737 \\
\hline $\mathrm{TSH}$ & $1.90(1.588)$ & $1.95(2.175)$ & $1.84(0.845)$ & $1.93(1.495)$ & 0.551 \\
\hline Homocysteine & $13.53(4.762)$ & $13.18(3.996)$ & $13.51(5.559)$ & $14.19(4.266)$ & 0.551 \\
\hline $\mathrm{Ca}$ & $2.31(0.117)$ & $2.29(0.108)$ & $2.33(0.118)$ & $2.32(0.127)$ & 0.302 \\
\hline Cholesterol & $5.17(1.204)$ & $5.04(1.360)$ & $5.25(1.077)$ & $5.24(1.145)$ & 0.412 \\
\hline $\mathrm{HDL}$ & $1.59(0.553)$ & $1.48(0.532)$ & $1.65(0.561)$ & $1.67(0.554)$ & 0.302 \\
\hline LDL & $3.01(1.006)$ & $2.99(1.147)$ & $3.00(0.909)$ & $3.05(0.933)$ & 0.737 \\
\hline$M_{C V}{ }^{a, b}$ & $90.60(5.830)$ & $88.73(5.898)$ & 91.36 (4.979) & 92.38 (6.399) & 0.000 \\
\hline GFR & $77.83(14.084)$ & $79.62(15.311)$ & $76.12(13.658)$ & $77.93(12.453)$ & 0.412 \\
\hline \multicolumn{6}{|l|}{ Comorbidities, \% } \\
\hline Depression & 41.0 & 35.5 & 37.5 & 57.4 & 0.126 \\
\hline Hypertension & 39.4 & 39.8 & 37.5 & 42.6 & 0.882 \\
\hline Heart failure & 14.3 & 18.3 & 14.4 & 7.4 & 0.390 \\
\hline Diabetes & 14.7 & 18.3 & 11.5 & 14.8 & 0.618 \\
\hline Liver failure & 1.6 & 3.2 & 1.0 & 0.0 & 0.390 \\
\hline Stroke & 9.6 & 9.7 & 8.7 & 11.1 & 0.882 \\
\hline \multicolumn{6}{|l|}{ Medications, \% } \\
\hline Antidepressants & 34.3 & 31.2 & 30.8 & 46.3 & 0.254 \\
\hline Antihypertensives & 43.8 & 48.4 & 43.3 & 37.0 & 0.560 \\
\hline NOACs & 7.6 & 8.6 & 8.7 & 3.7 & 0.560 \\
\hline Antiplatelets & 18.7 & 21.5 & 16.3 & 18.5 & 0.650 \\
\hline Neuroleptics & 5.2 & 4.3 & 2.9 & 11.1 & 0.254 \\
\hline Sleep pills & 10.8 & 11.8 & 6.7 & 16.7 & 0.257 \\
\hline Antianxietyc & 6.4 & 6.5 & 1.0 & 16.7 & 0.007 \\
\hline
\end{tabular}

Each beverage type was presented as a unit which corresponds to a Swedish standard drink which contains $12 \mathrm{gr}$ of pure alcohol ( $50 \mathrm{cl}$ low-strength alcohol beer, $33 \mathrm{cl}$ doublestrength bock beer, $12 \mathrm{cl}$ light wine, $8 \mathrm{cl}$ fortified wine, and $4 \mathrm{cl}$ spirits) using conversion factors based on average alcohol concentration by volume (beer $>3.5 \% 1 \mathrm{cl}=1 / 3 \mathrm{~g}$, wine $1 \mathrm{cl}=1 \mathrm{~g}$, spirits $1 \mathrm{cl}=3 \mathrm{~g}$ ). Weekly alcohol consumption is categorized as follows: (a) no alcohol consumption or less than one Swedish standard drink, without having former alcohol overconsumption (Group N), (b) 1 to 9 Swedish standard drinks for women and 1 to 14 Swedish standard drinks for men, without having former alcohol overconsumption (Group LM), and (c) > 9 Swedish standard drinks for women, $>14$ Swedish standard drinks for men, or/and former alcohol overconsumption according to their medical records (Group H). A 2-way ANCOVA was conducted to compare the main effects of alcohol consumption and education as well as their interaction effects on MMSE score. Age was included in the analysis as covariate. Adjusted means of MMSE scores of all groups are presented as adjusted means and standard errors. Covariates appearing in the model are evaluated at the following value: age $=62.18$. Continuous and interval data are presented as means and standard deviations, while categorical data are presented as percentages and frequencies except for the MRI rating scales which are presented as median and interquartile ranges. Differences in nominal variables were tested with the $x^{2}$ test. Likelihood ratio is presented when assumptions of $x^{2}$ test were violated. Differences in continuous variables were tested with the Kruskal-Wallis nonparametric test. All results and corresponding $p$ values are corrected by multiple comparisons, Benjamini-Hochberg procedure $(p<0.05)$ for each variable category separately. MMSE, Mini-mental State Examination; BMI, body mass index; MTA, medial temporal atrophy; GCA, global cortical atrophy; WMH, white matter hyperintensities; Na, Natrium; AST, aspartate transaminase; ALT, alanine transaminase; TSH, thyroid-stimulating hormone; Ca, calcium; HDL, high-density lipoprotein; LDL, low-density lipoprotein; GFR, glomerular filtration rate; MCV, mean

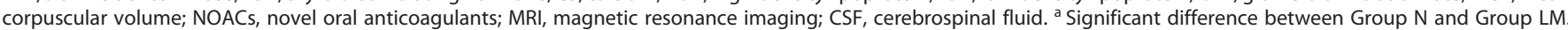
b Significant difference between Group N and Group H. ' Significant difference between Group LM and Group H. 
correlated with greater and quicker cognitive impairment in comparison with light to moderate alcohol intake [27]. There were no individuals at that high drinking level in our cohort. A possible explanation is that heavy drinkers are already hospitalized at that age or they refuse to undergo a structural cognitive assessment.

\section{Socioeconomic Factors}

Low to moderate alcohol consumption seems to be related to higher education (shown in online suppl. Fig. 1) which is a parameter of socioeconomic status and higher proportion of married individuals. Moreover, there are significantly less smokers among subjects with low to moderate consumption in comparison with heavy drinkers and they have significantly lower BMI and higher age in comparison with non- and rare drinkers, pointing to a healthier lifestyle. There are studies in the literature, suggesting that low to moderate consumption can be beneficial $[28,29]$, but it is unclear whether it is due to neuroprotective activity or related to the fact that low to moderate consumption is more commonly seen among subjects with higher socioeconomic status and better quality of life [30]. Nevertheless, it has been shown that non-drinkers have lower socioeconomic status [31,32].

\section{Comorbidity}

Alcohol consumption is not related to higher grade of cardiovascular or other relevant diseases, although alcohol overconsumption is usually comorbid with several diseases, especially psychiatric disorders [33]. This may be related to the fact that the consumption of alcohol was not high enough to negatively affect other organs. Our analysis showed higher usage of antianxiety medications among subjects with high alcohol consumption. It is known that heavy drinkers have a high prevalence of anxiety disorders [34]. Nevertheless, causal relationship is difficult to be explained as anxiety symptoms can be caused by the stress related to alcohol overconsumption, while some people use alcohol as a medication for their anxiety disorders [35]. Moreover, we found that never and rare drinkers had significantly lower levels of erythrocyte MCV. MCV is a laboratory marker which is usually increased among alcohol abusers [36], due to mechanisms related to the toxic activity of alcohol as well as the inadequate nutritional status which characterizes heavy drinkers [37] and can be used as a screening tool for identification of eventual former alcohol overconsumption [38]. Finally, no differences were identified regarding Alzheimer's-related biomarkers in CSF or grade of brain atrophy between the groups. Altered levels of those CSF

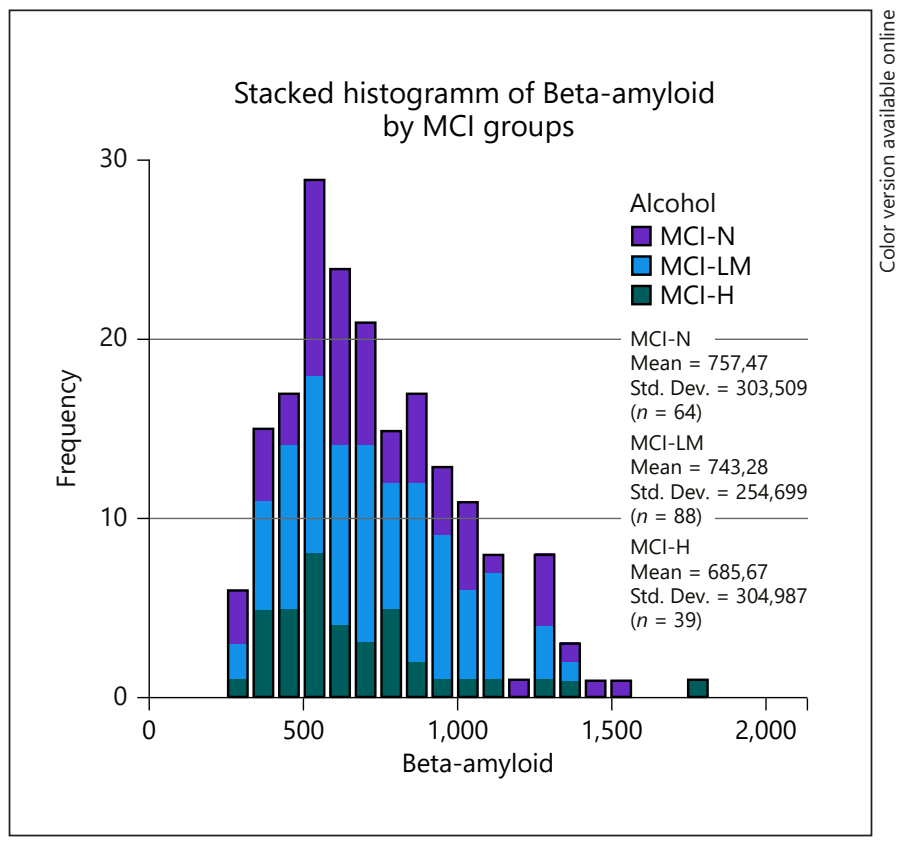

Fig. 2. Distribution of beta-amyloid. MCI, mild cognitive impairment.

biomarkers could point to prodromal $\mathrm{AD}$ and should be evident in a large group of MCI subjects. Our findings do not support the hypothesis that moderate alcohol consumption protects against development of $\mathrm{AD}$ since there were no significant differences in beta-amyloid levels between the groups (shown in Fig. 2). It would have been expected that the MCI cohort studied includes a large proportion of prodromal AD subjects showing lower levels of beta-amyloid.

Furthermore, we could not exclude subtle brain changes such as cortical thickness in certain areas since the MRI data were collected as rating scores from the routine evaluations. Further studies with more detailed imaging analysis would shed light on this question.

\section{Limitations}

Certain limitations should also be taken into consideration. Gender should be taken into account, as men and women differently metabolize alcohol $[39,40]$. We did not perform our analysis separately for men and women due to methodological reasons (low proportion of women in group with alcohol overconsumption), but we adopted the gender-specific Swedish guidelines. Second, the relatively small sample size limits the power of our study and increases the margin of error. In addition, the fact that level of alcohol consumption was self-reported can lead to recall bias. 
We have not estimated the genetic effects (APOE4) in our statistical analyses due to significantly few controllers having data on APOE4 in their medical records. Insufficient information about the controllers' drinking patterns and binge drinking induces also certain limitations in our study. Finally, we analyzed data from only one cognitive test (MMSE). Further analysis based on thorough and broad neuropsychological battery is needed.

\section{Conclusion}

According to our analysis, high alcohol consumption is not correlated with a more pronounced cognitive deficit or a distinct clinical severity at this early stage of cognitive impairment. Subjects with light to moderate consumption appear to have a better social background and a higher educational level. The limited sample size infers that the findings should be interpreted with caution. We are planning a follow-up study to ascertain if heavy drinkers have a different prognosis from those individuals without alcohol overconsumption.

\section{Statement of Ethics}

Ethical approval for our research project has been obtained (regional ethical review board in Stockholm, Diary No. 2016/164431). Patients have given written informed consent.

\section{Conflict of Interest Statement}

The authors have no conflicts of interest to declare.

\section{Funding Sources}

This study was supported by the Stockholm County Council (ALF funding), the Swedish Council for Information on Alcohol and Other Drugs (CAN), research funding Stockholm UniversityStockholm Region and FRF (Fonden för Rehabilitering och Medicinsk Forskning).

\section{Author Contributions}

T.T. collected and analyzed the data and wrote the manuscript. E.W. interpreted the data and contributed to the discussion. K.P. reviewed the statistical analysis and advised on methodology. O.L. analyzed and interpreted the data. A.B. analyzed the data and advised on methodology. D.R. interpreted the data and contributed to the discussion. L.-O.W. designed the study and analyzed and interpreted the data. All authors reviewed the manuscript and contributed to the manuscript's content.

\section{Data Availability Statement}

All data generated or analyzed during this study are included in this article and its online supplementary material files. Further enquiries can be directed to the corresponding author.

\section{References}

1 Harper C. The neurotoxicity of alcohol. Hum Exp Toxicol. 2007 Mar;26(3):251-7.

2 Zahr NM, Pfefferbaum A. Alcohol's effects on the brain: neuroimaging results in humans and animal models. Alcohol Res. 2017;38(2): 183-206.

3 Langballe EM, Ask H, Holmen J, Stordal E, Saltvedt I, Selbæk G, et al. Alcohol consumption and risk of dementia up to 27 years later in a large, population-based sample: the HUNT study, Norway. Eur J Epidemiol. 2015 Sep;30(9):1049-56.

4 Nordström P, Nordström A, Eriksson M, Wahlund LO, Gustafson Y. Risk factors in late adolescence for young-onset dementia in men: a nationwide cohort study. JAMA Intern Med. 2013 Sep 23;173(17):1612-8.

5 Kivipelto M, Solomon A. Alzheimer's disease - the ways of prevention. J Nutr Health Aging. 2008;12(1):89S-94S.

6 Oslin D, Atkinson RM, Smith DM, Hendrie H. Alcohol related dementia: proposed clinical criteria. Int J Geriatr Psychiatry. 1998 Apr; 13(4):203-12.
7 Sachdeva A, Chandra M, Choudhary M, Dayal P, Anand KS. Alcohol-related dementia and neurocognitive impairment: a Review Study. Int J High Risk Behav Addict. 2016 Sep; 5(3):e27976.

8 Ridley NJ, Draper B, Withall A. Alcohol-related dementia: an update of the evidence. Alzheimers Res Ther. 2013 Jan 25;5(1):3.

9 Kalinowski A, Humphreys K. Governmental standard drink definitions and low-risk alcohol consumption guidelines in 37 countries. Addiction. 2016;111:1293-8.

10 Kim JW, Lee DY, Lee BC, Jung MH, Kim H, Choi YS, et al. Alcohol and cognition in the elderly: a review. Psychiatry Investig. 2012 Mar;9(1):8-16.

11 Topiwala A, Allan CL, Valkanova V, Zsoldos E, Filippini N, Sexton C, et al. Moderate alcohol consumption as risk factor for adverse brain outcomes and cognitive decline: longitudinal cohort study. BMJ. 2017 Jun 6;357: j2353.
12 Ahlner F, Sigström R, Rydberg Sterner T, Mellqvist Fässberg M, Kern S, Östling S, et al. Increased alcohol consumption among Swedish 70-year-olds 1976 to 2016: analysis of data from the Gothenburg H70 birth cohort studies, Sweden. Alcohol Clin Exp Res. 2018 Dec; 42(12):2403-12.

13 Alcoholism USD of HAHSNI of HNI on AAA; US Department of Health and Human Services; National Institutes of Health; National Institute on Alcohol Abuse and Alcoholism. Helping patients who drink too much: a clinician's guide [Internet]. PsycEXTRA Dataset. 2005. Available from:

14 Anstey KJ, Mack HA, Cherbuin N. Alcohol consumption as a risk factor for dementia and cognitive decline: meta-analysis of prospective studies. Am J Geriatr Psychiatry. 2009 Jul; 17(7):542-55.

15 Ruitenberg A, van Swieten JC, Witteman JC, Mehta KM, van Duijn CM, Hofman A, et al. Alcohol consumption and risk of dementia: the Rotterdam Study. Lancet. 2002 Jan 26; 359(9303):281-6. 
16 Petersen RC. Mild cognitive impairment as a diagnostic entity. J Intern Med. 2004 Sep; 256(3):183-94.

17 Petersen RC, Doody R, Kurz A, Mohs RC, Morris JC, Rabins PV, et al. Current concepts in mild cognitive impairment. Arch Neurol. 2001 Dec;58(12):1985-92.

18 Folstein MF. Mini-mental state: a practical method for grading the cognitive state of patients for the clinician. Pergamon Press; 1975. p. 10.

19 Reid MC, Maciejewski PK, Hawkins KA, Bogardus ST Jr. Relationship between alcohol consumption and Folstein mini-mental status examination scores among older cognitively impaired adults. J Geriatr Psychiatry Neurol. 2002;15:31-7.

20 Wahlund LO, Westman E, van Westen D, Wallin A, Shams S, Cavallin L, et al. Imaging biomarkers of dementia: recommended visual rating scales with teaching cases. Insights Imaging. $2017 \mathrm{Feb}$;8(1):79-90.

21 Barkhof F, Fox NC, Bastos-Leite AJ, Scheltens P. Neuroimaging in dementia [internet]. Berlín: Springer; 2011.

22 Scheltens P, Leys D, Barkhof F, Huglo D, Weinstein HC, Vermersch P, et al. Atrophy of medial temporal lobes on MRI in "probable" Alzheimer's disease and normal ageing: diagnostic value and neuropsychological correlates. J Neurol Neurosurg Psychiatry. 1992;55: 967-72.

23 Ferreira D, Cavallin L, Larsson EM, Muehlboeck JS, Mecocci P, Vellas B, et al. Practical cut-offs for visual rating scales of medial temporal, frontal and posterior atrophy in $\mathrm{Alz}-$ heimer's disease and mild cognitive impairment. J Intern Med. 2015 Sep;278(3):277-90.
24 Pasquier F, Leys D, Weerts JG, Mounier-Vehier F, Barkhof F, Scheltens P. Inter- and intraobserver reproducibility of cerebral atrophy assessment on MRI scans with hemispheric infarcts. Eur Neurol. 1996;36(5): $268-72$.

25 Fazekas F, Chawluk JB, Alavi A, Hurtig HI, Zimmerman RA. MR signal abnormalities at $1.5 \mathrm{~T}$ in Alzheimer's dementia and normal aging. AJR Am J Roentgenol. 1987 Aug;149(2): 351-6.

26 Swedish National Board of Health and Welfare (2012). Available at: https://roi.socialstyrelsen.se/kvalitetsindikatorer/ej-langreriskbruk-av-alkohol/cfa9f2f6-25a4-4928bc23-66fble468e55. Accessed Januari 27.2021.

27 Sabia S, Elbaz A, Britton A, Bell S, Dugravot A, Shipley M, et al. Alcohol consumption and cognitive decline in early old age. Neurology. 2014 Jan 28;82(4):332-9.

28 Rubin E. To drink or not to drink: that is the question. Alcohol Clin Exp Res. 2014;38: 2889-92.

29 Zhang R, Shen L, Miles T, Shen Y, Cordero J Qi Y, et al. Association of low to moderate alcohol drinking with cognitive functions from middle to older age among US adults. JAMA Netw Open. 2020;3:e207922.

30 Towers A, Philipp M, Dulin P, Allen J. The "health benefits" of moderate drinking in older adults may be better explained by socioeconomic status. J Gerontol B Psychol Sci Soc Sci. 2018 Apr 16;73(4):649-54

31 Fillmore KM, Golding JM, Graves KL, Kniep S, Leino EV, Romelsjo A, et al. Alcohol consumption and mortality. I. Characteristics of drinking groups. Addiction. 1998;93:183203.
32 Fekjaer HO. Alcohol-a universal preventive agent? Addiction. 2013;108:2051-7.

33 Yang $\mathrm{P}$, Tao R, He C, Liu S, Wang Y, Zhang $\mathrm{X}$. The risk factors of the alcohol use disorders-through review of its comorbidities. Front Neurosci. 2018 May 11;12:303.

34 Gimeno C, Dorado ML, Roncero C, Szerman N, Vega P, Balanzá-Martínez V, et al. Treatment of comorbid alcohol dependence and anxiety disorder: review of the scientific evidence and recommendations for treatment. Front Psychiatry. 2017 Sep 22;8:173.

$35 \mathrm{McHugh}$ RK. Treatment of co-occurring anxiety disorders and substance use disorders. Harv Rev Psychiatry. 2015 Mar-Apr;23(2): 99-111.

36 Sillanaukee P. Laboratory markers of alcohol abuse. Alcohol Alcohol. 1996;31(6):613-6.

37 Ballard HS. The hematological complications of alcoholism. Alcohol Health Res World. 1997;21(1):42-52.

38 Mundle G, Ackermann K, Munkes J, Steinle $\mathrm{D}$, Mann K. Influence of age, alcohol consumption and abstinence on the sensitivity of carbohydrate-deficient transferrin, gammaglutamyl transferase and mean corpuscular volume. Alcohol Alcohol. 1999;34:760-6. Available from:

39 Erol A, Karpyak VM. Sex and gender-related differences in alcohol use and its consequences: contemporary knowledge and future research considerations. Drug Alcohol Depend. 2015;156:1-13

40 Graham K, Wilsnack R, Dawson D, Vogeltanz $\mathrm{N}$. Should alcohol consumption measures be adjusted for gender differences? Addiction. 1998;93:1137-47. 